International Journal of Pure and Applied Mathematics

Volume 84 No. 1 2013, 159-169

ISSN: 1311-8080 (printed version); ISSN: 1314-3395 (on-line version)

url: http://www.ijpam.eu

doi: http://dx.doi.org/10.12732/ijpam.v84i1.12

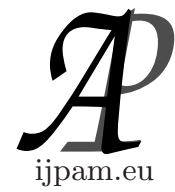

\title{
THE $w$-MODULAR FUNCTION AND THE EVALUATION OF ROGERS RAMANUJAN CONTINUED FRACTION
}

\author{
Nikolaos Bagis \\ Department of Informatics \\ Aristotle University of Thessaloniki \\ Thessaloniki, Greece
}

\begin{abstract}
In previous article we have considered attached to elliptic singular moduli $k_{r}$, the parameter $w_{r}$, in which if one knows its value then can evaluate explicit the elliptic singular moduli and the fifth degree singular moduli $k_{25 r}$ in radicals. In the present article we give modular equations of this parameter $w_{r}$ and a table of some lower values and use this parameter to solve the RogersRamanujan continued fraction.
\end{abstract}

AMS Subject Classification: 11Y65, 11A55, 35J75, 33E05

Key Words: modular equations, singular moduli, continued fractions, Ramanujan, algebraic equations, algebraic numbers, elliptic functions

\section{Introduction}

In $([2],[3],[5])$ we have considered the function $w_{r}=\sqrt{k_{r} k_{25 r}}$. Also it have been proved that if one knows $w_{r}$ then can find in radicals the value of $k_{r}$ and $k_{25 r}$.

Values of $k_{25 r}$ are useful in the construction of $\pi$ formulas as also for the evaluation of the Rogers-Ramanujan continued fraction-(RRCF). Here we consider the case of RRCF.

Ramanujan was the first who consider and study systematically these theories. For the Ramanujan's results one can see [8], [9], [10], [11].

Received: January 14, 2013

(c) 2013 Academic Publications, Ltd. url: www.acadpubl.eu 
We note to the unfamiliar reader that the elliptic integral of the first kind $K$ and the singular moduli $k_{r}$ are defined respectively as (see [1],[19],[16])

$$
K(x)=\int_{0}^{\pi / 2} \frac{d t}{\sqrt{1-x^{2} \sin (t)^{2}}}=\frac{\pi}{2}{ }_{2} F_{1}\left(\frac{1}{2}, \frac{1}{2} ; 1 ; x^{2}\right)
$$

and

$$
\frac{K\left(\sqrt{1-k_{r}^{2}}\right)}{K\left(k_{r}\right)}=\sqrt{r}
$$

also when $r$ is positive rational then $k_{r}$ is algebraic.

The RRCF is defined as

$$
R(q)=\frac{q^{1 / 5}}{1+} \frac{q}{1+} \frac{q^{2}}{1+} \frac{q^{3}}{1+} \ldots
$$

where $q=e^{-\pi \sqrt{r}}, r>0$. For this it have been proved that (see [2],[5]):

$$
R\left(q^{2}\right)^{-5}-11-R\left(q^{2}\right)^{5}=\frac{\left(k_{r} k_{r}^{\prime}\right)^{2}}{\left(w_{r} w_{r}^{\prime}\right)^{2}}\left(\frac{w_{r}}{k_{r}}+\frac{w_{r}^{\prime}}{k_{r}^{\prime}}-\frac{w_{r} w_{r}^{\prime}}{k_{r} k_{r}^{\prime}}\right)^{3}
$$

Hence evaluating $R(q)$ it is better to know $w_{r}$.

For the case of $\pi$ formulas except of $k_{r}$ we need and another function called elliptic alpha function $a(r)$ which have been defined and treated in [14] see also [6]. The evaluation of $k_{r}$ and $k_{25 r}$, can formulated as follows (see [2], [3], [5]).

Assume that we know $w=w_{r}$, then solve the equation

$$
w=\sqrt{\frac{L(18+L)}{6(64+3 L)}}
$$

then set

$$
M=\frac{18+L}{64+3 L}
$$

It have been shown that

$$
\begin{gathered}
\frac{\sqrt{k_{25 r}}}{\sqrt{w}}=\frac{\sqrt{w}}{\sqrt{k_{r}}}=\frac{1}{2} \sqrt{4+\frac{2}{3}\left(\frac{L^{1 / 6}}{M^{1 / 6}}-4 \frac{M^{1 / 6}}{L^{1 / 6}}\right)^{2}}+ \\
+\frac{1}{2} \sqrt{\frac{2}{3}}\left(\frac{L^{1 / 6}}{M^{1 / 6}}-4 \frac{M^{1 / 6}}{L^{1 / 6}}\right)
\end{gathered}
$$




\section{Lower Modular Equations of $w$}

We set the function $A(x)$ as

$$
A(w):=\frac{S}{\sqrt{6}}+\frac{\sqrt{4+\frac{2}{3} S^{2}}}{2}
$$

where

$$
S=\frac{L^{1 / 6}}{M^{1 / 6}}-4 \frac{M^{1 / 6}}{L^{1 / 6}}
$$

and the $L, M$ are that of (5) and (6).

Hence

$$
k_{r}=\frac{w_{r}}{A\left(w_{r}\right)^{2}}
$$

Theorem 1. The modular equations who relates $w_{4 r}$ with $w_{r}$ (duplication formula) are

$$
\begin{gathered}
w_{4 r}=\sqrt{\frac{\left(1-\sqrt{1-\frac{w_{r}^{2}}{A\left(w_{r}\right)^{4}}}\right)\left(1-\sqrt{1-A\left(w_{r}\right)^{4} w_{r}^{2}}\right)}{\left(1+\sqrt{1-\frac{w_{r}^{2}}{A\left(w_{r}\right)^{4}}}\right)\left(1+\sqrt{1-A\left(w_{r}\right)^{4} w_{r}^{2}}\right)}} \\
w_{r / 4}=\frac{4 A\left(w_{r}\right) \sqrt{w_{r}}}{A\left(w_{r}\right)+\sqrt{w_{r}}+A\left(w_{r}\right)^{2} \sqrt{w_{r}}+A\left(w_{r}\right) w_{r}} .
\end{gathered}
$$

Proof.

$$
w_{4 r}=\sqrt{k_{4 r} k_{100 r}}
$$

but

$$
k_{4 r}=\frac{1-k_{r}^{\prime}}{1+k_{r}^{\prime}}
$$

Hence

$$
w_{4 r}^{2}=\frac{1-k_{r}^{\prime}-\frac{\left(w_{r}^{\prime}\right)^{2}}{k_{r}^{\prime}}+w_{r}^{\prime}}{1+k_{r}^{\prime}+\frac{\left(w_{r}^{\prime}\right)^{2}}{k_{r}^{\prime}}+w_{r}^{\prime}}
$$

hence using (10) we get (11). For to prove (12) we have

$$
k_{r}=\frac{4 \sqrt{k_{4 r}}}{\left(1+\sqrt{k_{4 r}}\right)^{2}}
$$

From

$$
w_{r}^{2}=k_{r} k_{25 r}
$$


using (15) and (10) in (17) we get the desired result.

For the fifth degree modular equation of $w_{r}$ we have the next

Theorem 2.

$$
\begin{aligned}
\left(w_{25 r} w_{25 r}^{\prime}\right)^{2}= & \frac{w_{r}^{2}\left(A\left(w_{r}\right)^{4}-w_{r}^{2}\right)}{A\left(w_{r}\right)^{8}} H\left(\frac{A\left(w_{r}\right)^{8}\left(w_{r} w_{r}^{\prime}\right)^{2}}{w_{r}^{2}\left(A\left(w_{r}\right)^{4}-w_{r}^{2}\right)}\right)^{2} \times \\
& \times H\left(\frac{w_{r}^{2}\left(A\left(w_{r}\right)^{4}-w_{r}^{2}\right)}{A\left(w_{r}\right)^{8}\left(w_{r / 25} w_{r / 25}^{\prime}\right)^{2}}\right) \cdot
\end{aligned}
$$

Proof. If $r \in \mathbf{R}_{+}^{*}$ set $H$ to be the function (see relation (20) below), such that

$$
\frac{k_{25 r} k_{25 r}^{\prime}}{k_{r} k_{r}^{\prime}}=H\left(\frac{k_{r} k_{r}^{\prime}}{k_{r / 25} k_{r / 25}^{\prime}}\right)
$$

Then

$$
\begin{gathered}
\left(w_{25 r} w_{25 r}^{\prime}\right)^{2}=\frac{k_{25^{2} r} k_{25{ }^{2} r}^{\prime} k_{25 r} k_{25 r}^{\prime}}{k_{25 r} k_{25 r}^{\prime} k_{r} k_{r}^{\prime}} k_{25 r} k_{25 r}^{\prime} k_{r} k_{r}^{\prime}= \\
=k_{25 r} k_{25 r}^{\prime} k_{r} k_{r}^{\prime} H\left(\frac{k_{25 r} k_{25 r}^{\prime}}{k_{r} k_{r}^{\prime}}\right) H\left(\frac{k_{r} k_{r}^{\prime}}{k_{r / 25} k_{r / 25}^{\prime}}\right)= \\
=\frac{k_{25 r} k_{25 r}^{\prime}}{k_{r} k_{r}^{\prime}}\left(k_{r} k_{r}^{\prime}\right)^{2} H\left(\frac{\left(w_{r} w_{r}^{\prime}\right)^{2}}{\left(k_{r} k_{r}^{\prime}\right)^{2}}\right) H\left(\frac{\left(k_{r} k_{r}^{\prime}\right)^{2}}{\left(w_{r / 25} w_{r / 25}^{\prime}\right)^{2}}\right)= \\
=\left(k_{r} k_{r}^{\prime}\right)^{2} H\left(\frac{\left(w_{r} w_{r}^{\prime}\right)^{2}}{\left(k_{r} k_{r}^{\prime}\right)^{2}}\right)^{2} H\left(\frac{\left(k_{r} k_{r}^{\prime}\right)^{2}}{\left(w_{r / 25} w_{r / 25}^{\prime}\right)^{2}}\right)
\end{gathered}
$$

But

$$
\left(k_{r} k_{r}^{\prime}\right)^{2}=\frac{w_{r}^{2}\left(A\left(w_{r}\right)^{4}-w_{r}^{2}\right)}{A\left(w_{r}\right)^{8}}
$$

From the above we get the desired result. The function $H$ in view of [4] is given as

$$
H(x)=U\left(Q\left(U^{*}\left(x^{1 / 6}\right)^{6}\right)^{1 / 6}\right)^{6}
$$

where

$$
Q(x)=\frac{\left(-1-e^{\frac{1}{5} y}+e^{\frac{2}{5} y}\right)^{5}}{\left(e^{\frac{1}{5} y}-e^{\frac{2}{5} y}+2 e^{\frac{3}{5} y}-3 e^{\frac{4}{5} y}+5 e^{y}+3 e^{\frac{6}{5} y}+2 e^{\frac{7}{5} y}+e^{\frac{8}{5} y}+e^{\frac{9}{5} y}\right)}
$$


and

$$
\begin{gathered}
y=\operatorname{arcsinh}\left(\frac{11+x}{2}\right) . \\
U(x)=\sqrt{-\frac{5}{3 x^{2}}+\frac{25}{3 x^{2} h(x)}+\frac{x^{4}}{h(x)}+\frac{h(x)}{3 x^{2}}}
\end{gathered}
$$

where

$$
\begin{gathered}
h(x)=\left(-125-9 x^{6}+3 \sqrt{3} \sqrt{-125 x^{6}-22 x^{12}-x^{18}}\right)^{1 / 3} \\
U^{*}(x)=\sqrt{-\frac{1}{2 x^{2}}+\frac{x^{4}}{2}+\frac{\sqrt{1+18 x^{6}+x^{12}}}{2 x^{2}}} .
\end{gathered}
$$

In general assume that we have an arbitrary $n$-th degree modular equation of the singular moduli $k_{r}$, such that

$$
f\left(k_{r}, k_{n^{2} r}\right)=\text { const, } \forall r>0
$$

then

$$
f\left(k_{25 r}, k_{25 n^{2} r}\right)=\mathrm{const}
$$

using now (10) we get

$$
\begin{gathered}
f\left(\frac{w_{r}^{2} A\left(w_{r}\right)^{2}}{w_{r}}, \frac{w_{n^{2} r}^{2} A\left(w_{n^{2} r}\right)^{2}}{w_{n^{2} r}}\right)=\mathrm{const} \\
f\left(w_{r} A\left(w_{r}\right)^{2}, w_{n^{2} r} A\left(w_{n^{2} r}\right)^{2}\right)=\mathrm{const}
\end{gathered}
$$

Hence

Theorem 3. If the $n$-th degree modular equation of $k_{r}$ is

$$
f\left(k_{r}, k_{n^{2} r}\right)=\text { const }
$$

then

$$
f\left(w_{r} A\left(w_{r}\right)^{2}, w_{n^{2} r} A\left(w_{n^{2} r}\right)^{2}\right)=\text { const }
$$

and

$$
f\left(\frac{w_{r}}{A\left(w_{r}\right)^{2}}, \frac{w_{n^{2} r}}{A\left(w_{n^{2} r}\right)^{2}}\right)=\text { const. }
$$

Theorem 3 gives us the general case of the reduction of a modular equation of $k_{r}$ to that of $w_{r}$. As someone can see it is very complicated taking in mind that we have the argument of $w_{n^{2} r}$ inside $A(x)$. However there exist a straight 
forward evaluation, if one knows the solution of either side of $k_{r}$ or $k_{n^{2} r}$. More precisely we have

Theorem 4. Suppose that

$$
k_{n^{2} r}=f\left(k_{r}\right)
$$

then

$$
w_{n^{2} r}^{2}=f\left(w_{r} A\left(w_{r}\right)\right) f\left(\frac{w_{r}}{A\left(w_{r}\right)^{2}}\right) .
$$

Proof. From (32) we get

$$
k_{25 n^{2} r}=f\left(k_{25 r}\right)
$$

Hence

$$
\frac{w_{n^{2} r}^{2}}{k_{n^{2} r}}=f\left(\frac{w_{r}^{2}}{k_{r}}\right)
$$

or

$$
w_{n^{2} r}^{2}=f\left(\frac{w_{r}^{2}}{k_{r}}\right) k_{n^{2} r}
$$

or

$$
w_{n^{2} r}^{2}=f\left(\frac{w_{r}^{2} A\left(w_{r}\right)^{2}}{w_{r}}\right) f\left(k_{r}\right)
$$

or

$$
w_{n^{2} r}^{2}=f\left(w_{r} A\left(w_{r}\right)\right) f\left(\frac{w_{r}}{A\left(w_{r}\right)^{2}}\right)
$$

which is the desired result.

As application one can get the evaluation of $w_{9 r}$ which follows from the solvable equation (see [10], [3]):

$$
\sqrt{k_{r} k_{9 r}}+\sqrt{k_{r}^{\prime} k_{9 r}^{\prime}}=1
$$

or equivalently

$$
\begin{gathered}
u^{4}-256 u v+384 u^{2} v-132 u^{3} v+384 u v^{2}-762 u^{2} v^{2}+384 u^{3} v^{2}- \\
-132 u v^{3}+384 u^{2} v^{3}-256 u^{3} v^{3}+v^{4}=0
\end{gathered}
$$

where $u=k_{9 r}^{2}, v=k_{r}^{2}$.

We call the solution of (35) T, then $u=T(v)$ and form Theorem 4 we get

Theorem 5. If $f(x)=\sqrt{T\left(x^{2}\right)}$ then

$$
w_{9 r}^{2}=f\left(w_{r} A\left(w_{r}\right)\right) f\left(\frac{w_{r}}{A\left(w_{r}\right)^{2}}\right) .
$$




\section{The Rogers-Ramanujan Continued Fraction and the $w$-Moduli}

From the above discussion and identities (4),(7),(8) and (10) it is clear that $\mathrm{RRCF}$ can be put in the form

$$
R\left(q^{2}\right)^{-5}-11-R\left(q^{2}\right)^{5}=G\left(w_{r}\right),
$$

Hence it is better to describe $R(q)$ using the $w$-moduli and not the classical elliptic singular moduli $k_{r}$.

Theorem 6. If $q=e^{-\pi \sqrt{r}}$, then

$$
R\left(q^{2}\right)^{-5}-11-R\left(q^{2}\right)^{5}=\frac{\left(\sqrt{A\left(w_{r}\right)^{4}-w_{r}^{2}}+w_{r}^{\prime}-A\left(w_{r}\right)^{2} w_{r}^{\prime}\right)^{3}}{\left(A\left(w_{r}\right) w_{r}^{\prime}\right)^{2} \sqrt{A\left(w_{r}\right)^{4}-w_{r}^{2}}}
$$

and

$$
w_{r}^{\prime}=\sqrt{k_{r}^{\prime} k_{25 r}^{\prime}}=\sqrt{\sqrt{1-\frac{w_{r}^{2}}{A\left(w_{r}\right)^{4}}} \sqrt{1-A\left(w_{r}\right)^{4} w_{r}^{2}}} .
$$

As someone can see the modular equations of the above theorems, for example that of two degree, are very complicated. They give us the desired result, but the output is a rough evaluation rather an elegant simplified form. In literature the existing evaluations are special values and not straight forward general evaluations. One can compare them with the modular equations given by Ramanujan for the RRCF (see [10],[11],[13]), which are also, give not elegant results.

Note also that the evaluation (18) of $w_{25 r}$ as also of $w_{4 r}$ and $w_{r / 4}$ can be done using existing equations. For example to evaluate $w_{4 r}=\sqrt{k_{4 r} k_{100 r}}$ we first evaluate $k_{4 r}$ and $k_{100 r}$, then from (14) we evaluate $w_{4 r}$. But this happens only if we know $k_{r}$ and $k_{25 r}$. Here with the knowledge of one value only (that of $w_{r}$ ) we evaluate RRCF successfully and both $k_{r}$ and $k_{25 r}$.

\section{Table of $w_{r}$}

For $r=2 / 35$

$$
w_{2 / 35}=-168+45 \sqrt{14}+5 \sqrt{2245-600 \sqrt{14}}
$$


For $r=3 / 20$

$$
w_{3 / 20}=2 \sqrt{-8304+2626 \sqrt{10}-2 \sqrt{30(1149197-363408 \sqrt{10})}}
$$

For $r=13 / 10$

$$
\begin{gathered}
w_{13 / 10}^{2}=-12 \sqrt{15673137249530-1374625071240 \sqrt{130}}+ \\
+34(-988019+86655 \sqrt{130})
\end{gathered}
$$

For $r=7 / 10$

$$
\frac{w_{7 / 10}=}{\sqrt{-108096+28890 \sqrt{14}-2 \sqrt{35(166925663-44612760 \sqrt{14})}}}
$$

For $r=3 / 10$

$$
w_{3 / 10}=\sqrt{-416+170 \sqrt{6}-2 \sqrt{86565-35340 \sqrt{6}}}
$$

For $r=1 / 30$

$$
w_{1 / 30}=\sqrt{2(-208+85 \sqrt{6}+\sqrt{86565-35340 \sqrt{6}})}
$$

For $r=1 / 25$

$$
w_{1 / 25}=\sqrt{-3+\frac{3 \sqrt{5}}{2}+\sqrt{-20+9 \sqrt{5}}}
$$

For $r=1 / 20$

$$
w_{1 / 20}=2 \sqrt{-14-6 \sqrt{5}+\sqrt{380+170 \sqrt{5}}}
$$

For $r=1 / 15$

$$
w_{1 / 15}=\frac{\sqrt{4+\sqrt{15}}}{4}
$$

For $r=1 / 10$

$$
w_{1 / 10}=\sqrt{2(-3+\sqrt{10})}
$$


For $r=1 / 5$

$$
w_{1 / 5}=\sqrt{\frac{1}{2}(-2+\sqrt{5})}
$$

For $r=2 / 5$

$$
w_{2 / 5}=-3+\sqrt{10}
$$

For $r=3 / 5$

$$
w_{3 / 5}=\frac{\sqrt{4-\sqrt{15}}}{4}
$$

For $r=4 / 5$

$$
w_{4 / 5}=5+2 \sqrt{5}-2 \sqrt{11+5 \sqrt{5}}
$$

For $r=5 / 5=1$

$$
w_{1}=\frac{1}{\sqrt{2}(51841+23184 \sqrt{5}+12 \sqrt{37325880+16692641 \sqrt{5}})^{1 / 4}}
$$

For $r=6 / 5$

$$
w_{6 / 5}=-12+5 \sqrt{6}-\sqrt{5(49-20 \sqrt{6})}
$$

For $r=8 / 5$

$$
w_{8 / 5}=25+8 \sqrt{10}-4 \sqrt{79+25 \sqrt{10}}
$$

For $r=9 / 5$

$$
w_{9 / 5}=25+8 \sqrt{10}-4 \sqrt{79+25 \sqrt{10}}
$$

For $r=11 / 5$

$$
w_{11 / 5}=\frac{1}{4 \sqrt{1194+360 \sqrt{11}+\sqrt{2851435+859740 \sqrt{11}}}}
$$

For $r=12 / 5$

$$
w_{12 / 5}=95-30 \sqrt{10}-2 \sqrt{6(721-228 \sqrt{10})}
$$

For $r=15 / 5=3$

$$
w_{14 / 5}=-168+45 \sqrt{14}-5 \sqrt{2245-600 \sqrt{14}}
$$


For $r=17 / 5$

$$
w_{17 / 5}=\sqrt{-41+\frac{9 \sqrt{85}}{2}-6 \sqrt{5(-378+41 \sqrt{85})}}
$$

For $r=18 / 5$

$$
w_{18 / 5}=u
$$

where

$$
1 / u-u=2(240+98 \sqrt{6}-5 \sqrt{5(921+376 \sqrt{6})})
$$

For $r=20 / 5=4$

$$
\begin{gathered}
v+1 / v=6(69121+30912 \sqrt{5}+16 \sqrt{37325880+16692641 \sqrt{5}}) \\
w_{4}=\sqrt{(3-2 \sqrt{2}) v}
\end{gathered}
$$

\section{References}

[1] J.V. Armitage, W.F. Eberlein, Elliptic Functions, Cambridge University Press (2006).

[2] N.D. Bagis, Parametric evaluations of the Rogers Ramanujan continued fraction, International Journal of Mathematics and Mathematical Sciences (2011).

[3] N.D. Bagis, The complete evaluation of Rogers-Ramanujan and other continued fractions with elliptic functions, ArXiv: 1008.1304v1 [math.GM] (2010).

[4] N.D. Bagis, Evaluation of fifth degree elliptic singular moduli, ArXiv: 1202.6246v2 [math.GM] (2012).

[5] N.D. Bagis, On a general sextic equation solved by the Rogers-Ramanujan continued fraction, Arxiv: 1111.6023v2 [math.GM] (2012).

[6] N.D. Bagis, M.L. Glasser, Conjectures on the evaluation of alternative modular bases and formulas approximating $1 / \pi$, Journal of Number Theory, Elsevier (2012). 
[7] N.D. Bagis, A general method for constructing Ramanujan formulas for $1 / \pi^{\nu}$, The Mathematica Journal (2013), To Appear.

[8] B.C. Berndt, Ramanujan's Notebooks Part I, Springer Verlag, New York (1985).

[9] B.C. Berndt, Ramanujan's Notebooks Part II, Springer Verlag, New York (1989).

[10] B.C. Berndt, Ramanujan's Notebooks Part III, Springer Verlag, New York (1991).

[11] B.C. Berndt, Ramanujan's Notebooks Part V, Springer Verlag, New York (1998).

[12] B.C. Berndt, H.H. Chan, Notes on Ramanujan's singular moduli, In: Proceedings of the Fifth Conference of the Canadian Number Theory Association (Ed-s: R. Gupta, K.S. Williams) (1998), 7-16

[13] B.C. Berndt, H.H. Chan, S.S. Huang, S.Y. Kang, J. Sohn, S.H. Son, The Rogers-Ramanujan continued fraction, J. Comput. Appl. Math., 105 (1999), 9-24

[14] J.M. Borwein, P.B. Borwein, Pi and the AGM, John Wiley and Sons, Inc. New York-Chichester-Brisbane-Toronto-Singapore (1987)

[15] J.M. Borwein, P.B. Borwein, D.H. Bailey, Ramanujan, modular equations, and approximations to $\mathrm{Pi}$ or how to compute one billion digits of $\mathrm{Pi}$, Amer. Math. Monthly, 96 (1989), 201-219

[16] D. Broadhurst, Solutions by radicals at singular values $k_{N}$ from new class invariants for $N \equiv 3 \bmod 8$, ArXiv: 0807.2976 [math-ph] (2008).

[17] W. Duke, Continued fractions and modular functions, Bull. Amer. Math. Soc. (N.S.), 42 (2005), 137-162.

[18] M. Trott, Modular equations of the Rogers-Ramanujan continued fraction, Mathematica Journal, 9 (2004), 314-333

[19] E.T. Whittaker, G.N. Watson, A Course on Modern Analysis, Cambridge University Press (1927). 
